\title{
Dysfonctions sexuelles masculines et rapport à l'autre. Perspectives sexoanalytiques
}

\author{
Denise MEDICO
}

Département de sexologie, Université du Québec, Montréal (Canada) Centre de Médecine de la Reproduction MEDIXY, Lausanne (Suisse)

RESUME

Selon la sexoanalyse, les troubles sexuels prennent un sens dans l'économie psychique et sexuelle de l'individu et c'est à travers sa compréhension qu'ils peuvent être pris en charge. Les dysfonctions sexuelles psychogènes sont, dans ce contexte, envisagées comme des manifestations particulières du rapport à l'Autre, un rapport - celui de la sexualité et de l'érotisme - dans lequel l'individu se trouve confronté à ses propres angoisses, défenses ou besoins. Trois vignettes cliniques d'hommes présentant des difficultés érectiles seront utilisées. Elles illustreront comment, au-delà d'un même critère diagnostique, se profilent des dynamiques sexuelles différentes au plan de la genralité, de la fantasmatique et du rapport à l'autre. Nous en concluons la nécessité d'élaborer une compréhension holistique et dynamique des troubles de la sexualité. Le travail thérapeutique devrait ainsi s'axer sur la santé sexuelle au sens large et non seulement sur une fonctionnalité physiologique standardisée et focalisée sur le symptôme.

Mots clés : dysfonctions sexuelles, psychogène, masculinité, sexoanalyse, psychothérapie, santé sexuelle

\section{INTRODUCTION}

Que peut-on faire pour les personnes se présentant avec des dysfonctions sexuelles psychogènes, situationnelles ou relationnelles? Les essais cliniques sur l'efficacité des traitements pharmacologiques (notamment avec le sildénafil) sur les troubles érectiles psychogènes semblent concluants $[5,11,13]$. Il apparaît cependant au sexologue clinicien que le problème puisse être pensé autrement... un autrement que l'approche sexoanalytique se propose d'explorer en resituant le trouble dans la dynamique sexuelle et érotique ${ }^{1}$ de l'individu.

\section{LA SEXOANALYSE, QUELQUES HYPOTHĖSES}

La sexoanalyse [3] peut être définie comme une théorie du développement sexuel et une approche thérapeutique des désordres sexuels à travers la compréhension des significations inconscientes du trouble et un travail correctif sur l'imaginaire. Le sens du trouble est interprété en fonction de la dynamique érotique du patient, de son développement psychosexuel et surtout de l'analyse de sa fantasmatique (rêves et fantasmes érotiques) [1,12]. Sa spécificité au plan théorique et thérapeutique est d'étudier la genralité (sentiment de masculinité et de féminité) ${ }^{2}$ et le rapport à l'autre, notamment dans sa propre genralité. Pour la sexoanalyse, la sexualité est un construit psychique qui a essentiellement trois fonctions. La première est d'ordre hédonique, l'individu cherche à atteindre un plaisir et un sentiment de satisfaction. La deuxième est d'ordre complétive, dans la sexualité se jouent les besoins

Correspondance:

Dr Denise Medico - MEDIXY - 8, Av. Jomini, CH-1004 Lausanne, Suisse - Tel + 41(0)216410570 - Fax +41(0)21641 0571 -Email denise.medico@bluewin.ch 
fusionnels d'aimer et d'être aimé, ainsi que les sentiments de valorisation et de réassurance par rapport à l'identité de genre. Et la troisième est d'ordre défensive, car la sexualité peut également servir à masquer ou à résoudre provisoirement certains conflits comme des anxiétés d'abandon, des blessures narcissiques, des traumatismes sexuels ou des angoisses de mort.

Selon la théorie sexoanalytique, qui se base en partie sur les travaux de Stoller $[14,15,16]^{3}$, le garçon comme la fille passerait par un stade initial de féminité - appelé protoféminité 4 - dans lequel le petit enfant vit un moment de fusion et d'identification genrale à la mère. Cette protoféminité impliquera pour le garçon le besoin de se détacher de la mère et de la féminité pour atteindre un sentiment de masculinité [2]. Il y parvient grâce à l'agressivité phallique 5 qui facilitera la défusion d'avec la mère, l'individuation et le sentiment de masculinité.

Cette identification première à la mère va rendre le sentiment de masculinité des hommes plus fragile que ne peut l'être celui de féminité chez la femme. Le retour dans la fusion et la symbiose avec la mère (symbolique) reste une menace pour la masculinité et plusieurs anxiétés peuvent hanter l'espace intrapsychique inconscient : l'angoisse d'être féminisé, de perdre sa virilité ou son identité masculine, celle d'être réenglouti dans une féminité primaire, mais aussi des angoisses liées à la perte de la fusion avec la mère, des anxiétés d'abandon ou d'individuation. L'expérience clinique [3] et les travaux anthropologiques [8,9] semblent d'ailleurs concorder sur le sentiment de fragilité de la masculinité. Le rôle de l'agressivité phallique, qui "réfère à l'ensemble des conduites (fantasmatiques ou réelles) visant à démontrer la puissance virile et à imposer une domination intersexuelle et intrasexuelle" [3, p 59] est dès lors central dans le développement psychosexuel du garçon. Elle lui permettra par la suite de se positionner comme homme désirant et pénétrant dans sa sexualité.

\section{TROIS MANIFESTATIONS DE TROU- BLES ÉRECTILES}

Une carence, ou une absence d'investissement érotique, de l'agressivité phallique se répercute d'ailleurs souvent dans des troubles de la genralité ou des difficultés sexuelles, telles que les troubles érectiles. Comme le résume Crépault :

“Ce qui semble toutefois particulariser l'impuissant, c'est sa difficulté à érotiser l'agressivité phallique, du moins au plan de la réalité. Cette inaptitude semble davantage apparaître chez les hommes dont la personnalité comporte de fortes composantes fusionnelles, narcissiques ou paranoide" [3, p 222-223].

Pour illustrer la compréhension de cette difficulté (symptôme) développée par la sexoanalyse, nous utiliserons trois cas cliniques desquels nous tenterons de dégager la dynamique sexuelle et érotique. Nous y découvrirons trois rapports à l'autre marqués par la crainte, l'hostilité et l'anxiété - de perdre sa masculinité ou de ne pas être reconnu dans sa masculinité, d'être réenglouti dans le sexe de la femme, de détruire l'autre, de ne pas être aimé.

\section{L'autre clivé, fusionné ou souillé - le cas de Chris- tian6}

Christian, 31 ans, consulte pour des troubles érectiles avec sa partenaire. Il se présente comme un troubadour à la recherche de la pureté féminine et insiste à chaque séance sur ses caractéristiques artistiques, romantiques et féminines. Il dira également se sentir peu masculin. Il est attiré amoureusement par des femmes "pures"7, qui n'aiment pas la sexualité et avec qui il entretient un fantasme d'amour infini où "il doit la vénérer et lui faire l'amour comme s'il lui faisait des enfants". Cependant, il se sent (et l'est !) "impuissant" dans ces contextes fusionnels. Par contre, il se sent "viril" dans un contexte plus antifusionnel, lorsqu'il perçoit sa partenaire comme "une nymphomane" ou lorsqu'il est en colère et agressif. Il ressent alors un fort désir sexuel, une érection et une envie de pénétrer violemment sa partenaire.

1 Selon nous, la sexualité est un concept plus vaste que l'érotisme, qui réfère autant aux aspects génitaux, corporels, reproducteurs et identitaires de l'être humain conçu comme un être sexué. L'érotisme est un aspect de la sexualité dans laquelle celle-ci s'actualise dans une quête psychologique et souvent hédonique.

2 La masculinité et la féminité sont définies par Stoller [16] comme : "toute qualité ressentie comme masculine ou féminine par son possesseur" (p. 36). L'identité de genre se distingue du sexe biologique, car elle est un construit social et psychique renvoyant à un certain nombre de codes culturels et de sentiments d'appartenance à ces codes. L'identité de genre est un mélange de masculinité et de féminité, ce qui implique que l'on retrouve chez chacun des formes de masculinité et de féminité à des degrés différents.

3 Mais également, dans sa conception du développement psychosexuel, sur les travaux de Malher, de Mélanie Klein, ainsi que des travaux anthropologiques sur le développement de la masculinité comme ceux de Herdt et Stoller [15].

4 Ce concept central en sexoanalyse - la féminité primaire ou protoféminité - a d'abord été développé par Greenson [4] et Stoller [14, 16]. Ce dernier en dégage des implications au plan de l'identité de genre chez le garçons : "Le concept de noyau de l'identité de genre, cependant, modifie comme suit la théorie de Freud. Bien qu'il soit vrai que le premier amour du garçon soit hétérosexuel, et bien que les pères soient des rivaux trop puissants, il existe un stade plus précoce du développement de l'identité de genre dans lequel le garçon est fusionné avec sa mère [...] Selon la façon dont la mère laisse son fils se séparer d'elle et l'allure à laquelle elle le fait, cette phase de fusion avec elle laisse des effets résiduels qui peuvent s'exprimer comme des troubles de la masculinité" (p. 37). 
Christian a peur d'être abandonné et réduit au rang d'objet sexuel par la femme et pense que les femmes qui aiment le sexe pourraient le tromper. Malgré le désarroi qu'entraîne son sentiment d'impuissance, il y trouve une certain avantage, il lui permet de montrer aux femmes son idéal d'amour fusionnel et asexué. Il établit consciemment une étroite et ambivalente relation entre l'image de sa mère, décrite comme surprotectrice et insécure, et ses partenaires sexuelles. S'il peut "baiser virilement" des femmes qui lui font penser à sa mère, il décrit également la pénétration avec elles comme "le retour dans le vagin de ma mère, dans le ventre, pour ne pas mourir". Une image qu'il utilise lors de la masturbation semble réconcilier ces deux aspects de son érotisme : il imagine une jeune nonne vierge, réservée et pure. À l'idée de la transgression de cet interdit, celui de souiller la pureté féminine, il devient très excité.

La dynamique érotique de Christian s'ancre autour des notions de clivage entre deux images de la femme, la madone et la putain, qui parlent toutes deux de son rapport avec l'image de la symbiose première à la mère (protoféminité). Il porte son désir amoureux et fusionnel sur une femme pure et potentiellement mère. Mais cette femmemère réactive chez lui des angoisses de réengloutissement dans lesquelles il craint de se perdre, de perdre le peu de masculinité qu'il a acquis. Le trouble érectile lui permet de conserver le lien amoureux fusionnel à la mère et de se préserver de la crainte de grandir et d'être volé à sa mère. Une crainte qui se manifeste dans un rêve anxiogène récurrent, celui d'un voleur d'enfant fort et viril. Le malaise qu'il ressent lors de ce rêve est rapproché de la peur de ne pas avoir d'érections, il entraîne "la même douleur dans le ventre". Ce n'est qu'à travers l'impression de dégrader l'objet sexuel - pour ne pas être lui-même objet - qu'il peut se poser comme désirant et pénétrant, et vivre ainsi ses pulsions agressives face à sa mère captatrice. Des pulsions qui reflètent par ailleurs son désir d'individuation et de masculinisation. Le travail sexoanalytique avec Christian consiste à développer chez lui son agressivité phallique, lui permettant ainsi de rencontrer ses désirs d'individuation et un sentiment de masculinité plus solide. Ce qui l'amènera à ne plus craindre le rapprochement émotionnel avec la femme et avoir une érection dans ce contexte.

\section{L'autre à détruire et à protéger, le cas de Joël}

Joël est un homme de 41 ans, assez réservé et distant. Il consulte car il ne peut plus pénétrer sa femme qui vient de lui donner un enfant (par procréation assistée) et qui est décrite comme une mère et une épouse parfaite. Il expliquera plus précisément que, lors de la pénétration, il perd son érection après quelques secondes. Il dit être amoureux et être attiré par elle. Mais, par peur de l'échec, il évite tous contacts corporels qu'ils soient affectifs ou sexuels. Il se sent honteux et à peur que son épouse le quitte. En dehors cette relation, son érection et sa capacité à pénétrer sont intactes. Il perçoit également sa femme comme une victime - de son non désir - et ressent pour elle de la pitié, comme il en ressent pour sa mère qui était battue par son père. Les difficultés érectiles se sont aggravées lorsqu'une infection à une maladie sexuellement transmissible contractée dans une aventure extraconjugale a été décelée chez lui lors des investigations en PMA (procréation médicalement assistée).

Le travail d'analyse de l'inconscient sexuel de Joël mettra à jour de fortes composantes sadiques et destructrices, surtout dirigées envers la femme objet d'amour et l'image de la mère. Chez Joël, l'agressivité phallique se confond avec l'agressivité de destruction ${ }^{8}$. Il utilise (inconsciemment) le trouble érectile comme une défense, contre sa propre agressivité et contre les risques imaginaires d'endommager et de contaminer la femme. Les tentatives d'avoir un enfant et les nouveaux rôles parentaux semblent avoir fixé cette crainte inconsciente dans les schémas relationnels du couple parental de son enfance.

Par le travail sexoanalytique Joël va prendre conscience de l'hostilité et de la colère qui l'empêchent de faire l'amour à son épouse, une situation qui permet de la protéger (de ses désirs destructeurs) comme de la punir (en la privant de son désir et de contacts physiques). À partir de cette prise de conscience, des élaborations et des réappropriations fantasmatiques lui permettront de réinvestir érotiquement son objet d'amour.

\section{L'autre, menace ou renforcement narcissique, le cas de François}

François est jeune homme de 29 ans, séduisant, séducteur et se posant comme très masculin. Il consulte car il a peur

5 Voir Trempe [17] pour une critique du concept d'agressivité phallique et une comparaison entre les conceptions sexoanalytiques et psychanalytiques.

6 Afin de préserver l'anonymat, les noms cités ici sont fictifs.

7 Les mots entre guillemets sont des citations littérales du discours de la personne. 
de perdre sa capacité érectile étant donné que sa fonctionnalité sexuelle se restreint à un seul scénario : Il doit toujours commencer par donner un cunnilingus à sa partenaire et l'amener à l'orgasme, s'il perçoit que sa partenaire n'atteint pas l'orgasme, il "ne peut plus continuer", il perd son érection et le contexte érotique devient insupportable. Plusieurs craintes le hantent, celle de devenir impuissant, d'être désiré (séduit ?) par un homme et de ne pas être reconnu comme un mâle dominant par ses partenaires. Il veut comprendre pourquoi il est prisonnier de son scénario sexuel.

L'anxiété de masculinitude et le besoin d'être reconnu comme un homme sont très proches de la conscience de François. Il craint de ne pas être capable de remplir les exigences de la masculinité et de n'être pas aussi performant que son père. Face à ce sentiment fragile de masculinité, il cherche une réassurance dans la sexualité. Faire jouir la femme, c'est quelque part, triompher d'elle et pouvoir ainsi se poser comme homme dominant et pénétrant. Toute incertitude est alors vécue comme une menace qui le renvoie à cette anxiété, lui fait perdre son érection et lui donne envie de fuir. Au fond, François cherche surtout à être aimé.

\section{QUE RETENIR DU REGARD SEXOANA- LYTIQUE DANS LA PRISE EN CHARGE DES DYSFONCTIONS SEXUELLES ?}

Les troubles de l'érection, surtout lorsqu'ils sont psychogènes, sont à envisager comme un symptôme. Un symptôme qui sous-tend différentes significations intrapsychiques et différentes dynamiques érotiques que l'analyse de la complexité de la sexualité peut mettre à jour. Comme l'écrit Lépine, "la dysfonction érectile est un compromis qui permet de combattre des états émotifs pénibles afin de retrouver dans la réalité une manière de résoudre ses désirs sexuels et amoureux" [7, p 180]. En travaillant le sens profond et psychique du trouble, la sexoanalyse permet à l'individu de développer sa santé sexuelle.

Les approches de type mécanicistes qui ont pris une place importante dans la sexologie clinique contemporaine française proposent une vision de la sexualité souvent limitée à sa fonctionnalité. Une fonctionnalité qui est d'ailleurs ellemême réduite à l'expression du modèle théorique de la réponse sexuelle humaine tel qu'il a été dégagé par Master et Johnson ${ }^{9}$ [10] et qui décrit la sexualité dans une démarche linéaire, orientée vers un but, une décharge de tensions, un orgasme. Cependant, il conviendrait, pour ceux qui s'intéressent à la sexualité humaine et à l'érotisme, de ne pas perdre de vue que la sexualité peut également être envisagée dans une perspective holistique de le l'individu et de sa santé. Si les aspects fonctionnels et physiologiques de la réponse sexuelle sont centraux, la sexualité est également le lieu d'une recherche hédonique, de désirs de paternité (maternité), de l'expression de défenses et d'anxiétés profondes, d'attributs symboliques culturellement déterminés et surtout d'un rapport avec un autre être humain.

Qu'il soit imaginé ou réel, le rapport à l'Autre modifie, amplifie ou empêche l'expression du désir et sa potentialisation corporelle. La sexualité s'inscrit dans des rapports aux autres, où l'autre n'est jamais innocent dans le jeu des signifiants. Pour la comprendre il est central d'évaluer et comprendre la manière dont l'individu construit sa sexualité dans son rapport à l'autre, dont il l'intègre, comment il se positionne, ce qu'il recherche et ce qu'il craint de l'autre et de lui-même.

Il est ainsi important de resituer le sexuel dans l'économie psychique globale en visant la santé sexuelle à travers la satisfaction de la vie sexuelle dans son ensemble. Et non d'isoler le trouble sexuel - qui n'est au fond qu'un symptôme - dans une fonctionnalité mécaniciste, normative et pathologisante.

Dans cet article, nous avons présenté trois cas cliniques marqués par un rapport à l'autre ambivalent, emprunt de craintes et parfois d'hostilité. Comme le disait Stoller, l'érotisme et l'excitation se nourrissent de l'hostilité :

"Ma théorie est la suivante : en l'absence de facteurs physiologiques spéciaux (comme un accroissement soudain du taux d'androgènes chez l'homme ou chez la femme) et mis à part les effets évidents d'une stimulation des parties érogènes du corps, c'est l'hostilité - le désir manifeste ou masqué de nuire à l'autre - qui induit et renforce l'excitation sexuelle. L'absence d'hostilité engendre l'indifférence et l'ennui sexuels. Dans l'érotisme, l'hostilité est la tentative sans cesse renouvelée d'annuler les traumatismes et frustrations de l'enfance qui ont menacé le développement de la masculinité ou de la féminité. On trouve cette même dynamique, quoique à des doses et degrés différents, chez presque tous les individus - aussi bien ceux qui sont qualifiés de pervers que les autres" [14, p 20].

Le rapport à l'autre érotisé ne comporte-t-il pas toujours une certaine dose d'hostilité... et une certaine dose d'amour ? C'est entre ces deux pôles que va se situer la santé érotique et sexuelle que nous tentons de développer, ou de retrouver, chez nos patients.

8 L'agressivité destructrice se confond avec l'hostilité. En tant que manifestation de la haine, elle comporte un désir de faire mal, de causer du tort à autrui, de faire souffrir. La privation d'un besoin psycho-affectif, une dévalorisation narcissique ou un abandon par une personne significative peuvent entraîner un sentiment de haine et un désir de vengeance. [3, p 59].

9 Pour une analyse critique du modèle de la réponse sexuelle construit par Master et Johnson et de sa validité scientifique , voir [6]. 


\section{REFERENCES}

1. CRÉPAULT C. : L'imaginaire érotique et ses secrets. Sillery, Presses de l'Université du Québec, 1981.

2. CRÉPAULT C. : Protoféminité et développement sexuel. Essai sur l'ontogénèse sexuelle et ses vicissitudes. Sillery, Presses de l'Université du Québec, 1986.

3. CRÉPAULT C. : La sexoanalyse. Paris, Payot,1997.

4. GREENSON R.R. : Dis-identifying from mother : its special importance for the boy. Int. J. Psychoanal., 1968, $49: 370-374$.

5. JAROW J.P., BURNETT A.L., GERINGER A.M. : Clinical efficacy of sildenafil citrate based on etiology and response to prior treatment. J. Urol., 1999, 162 : 722-725.

6. TIEFER L.: Sex is not a natural act and other essays, 1995.

7. LÉPLNE J. : Le fantasme de la femme initiatrice chez les impuissants psychogènes secondaires. In : Crépault C., Côté $\mathrm{H}$. eds. Imaginaire et sexoanalyse ; explorations de l'inconscient sexuel. Montréal, Éditions I.R.I.S., 1999 : 173-182.

8. LÉVY J.J. : La sexoanalyse : quelques réflexions ethnosexologiques. In : Crépault C., Côté H. eds. Imaginaire et sexoanalyse ; explorations de l'inconscient sexuel. Montréal, Éditions I.R.I.S., 1999 : 37-50.

9. LÉVY J.J., CRÉPAULT C. : Une compréhension sexoanalytique du masculin dans les sociétés maghrébines. In : Crépault $\mathrm{C}$, Lévesque G. eds. Eros au féminin, éros au masculin. Nouvelles explorations en sexoanalyse. Sainte Foy, Presses du l'Université du Québec, 2001 : 173-184.

10. MASTERS W.H., JOHNSON V.E. : Human Sexual Response. Boston, Little \& Brown, 1966.

11. MONTORSI F., MCDERMOTT T.E., MORGAN R. et al. : Efficacy and safety of fixed-dose oral sildenafil in the treatment of erectile dysfunction of various etiologies. Urology, 1999, 53 : 1011-1018.

12. PASINI W., CRÉPAULT C. : L'imaginaire en sexologie clinique. Paris, Presses Universitaires de France, 1987.

13. OLSSON A.M., SPEAKMAN M.J., DINSMORE W.W., et al. : Sildenafil citrate (viagra) is effective and well tolerated for treating erectile dysfunction of psychogenic or mixed aetiology. Int. J. Clin. Pract., 2000, 54 : 561-566.

14. STOLLER R.J. : Sexual Excitement. New York. Pantheon Books, 1979.

15. STOLLER R.J., HERDT G.H. : The development of masculinity: a cross-cultural contribution. J. Am. Psychoanal. Assoc., $1982,30: 29-59$.

16. STOLLER R.J. : Masculin ou féminin ? Paris, Presses Universitaires de France, 1989. (version française de Presentation of Gender. New Haven, Yale University Press, 1985).

17. TREMPE J.P. : Le concept d'agressivité phallique dans la théorie sexoanalytique et psychanalytique. In : Crépault C., Lévesque G. eds. Eros au féminin, éros au masculin. Nouvelles explorations en sexoanalyse. Sainte Foy, Presses du l'Université du Québec, $2001: 25-44$.

\section{Sexoanalytical views on psychogenic male sexual dysfunction}

\section{Denise MEDICO}

According to sexoanalysis, sexual dysfunction has a specific significance in the individual's mental and sexual functioning and can be managed based on an understanding of these processes. Psychogenic sexual dysfunctions are considered to be particular manifestations of the relationship with the Other, a relationship, involving sexuality and eroticism, in which the individual is faced with his or her own anxieties, defences or needs. Three clinical cases in men with erectile dysfunction are presented. They illustrate how the same diagnostic criterion can correspond to various sexual dynamics in terms of general functioning, fantasies and the relationship with the other. The authors conclude on the need to develop a holistic and dynamic view of sexual dysfunction. Therapeutic management must be based on sexual health and not only a standardized physiological functionality focussed on the symptom.

Key-Words: sexual dysfunction, psychogenic, sexoanaIysis, psychotherapy, sexual health, masculinity 